\title{
Soft X-ray AGN luminosity function from ROSAT surveys
}

\section{Table of the binned soft X-ray luminosity function}

\author{
T. Miyaji ${ }^{1,2,3}$, G. Hasinger ${ }^{3}$, and M. Schmidt ${ }^{4}$ \\ 1 Department of Physics, Carnegie Mellon University, Pittsburgh, PA 15213, USA \\ 2 Max-Planck-Inst. für Extraterrestrische Physik, Postf. 1603, 85740 Garching, Germany \\ 3 Astrophysikalisches Institut Potsdam, An der Sternwarte 16, 14482 Potsdam, Germany \\ e-mail: ghasinger@aip.de \\ 4 California Institute of Technology, Pasadena, CA 91125, USA \\ e-mail: mxs@deimos.caltech.edu
}

Received 14 June 2000 / Accepted 8 January 2001

\begin{abstract}
This is the second paper of our investigation of the $0.5-2 \mathrm{keV}$ soft X-ray luminosity function (SXLF) of active galactic nuclei (AGN) using results from ROSAT surveys of various depth. The large dynamic range of the combined sample, from shallow large-area ROSAT All-Sky Survey (RASS)-based samples to the satellite's deepest pointed observation on the Lockman Hole, enabled us to trace the behavior of the SXLF. While the first paper (Miyaji et al. 2000, hereafter Paper I) emphasized the global behavior of the SXLF, cosmological evolution and contribution to the soft X-ray background, this paper presents actual numerical values for practical use of our results. To present the binned SXLF, we have used an improved estimator, which is free from biases associated with the conventional $\sum V_{\mathrm{a}}^{-1}$ estimator.
\end{abstract}

Key words. galaxies: active - galaxies: evolution - (galaxies:) quasars: general - X-rays: galaxies - X-rays: general

\section{Introduction}

The AGN/QSO luminosity function and its evolution with cosmic time are key observational quantities for understanding the origin of and accretion history onto supermassive black holes, which are now believed to occupy the centers of most galaxies. Since X-ray emission is one of the prominent characters of the AGN activity, X-ray surveys are efficient means of sampling AGNs for luminosity function and evolution studies. An X-ray selected sample of AGNs is particularly useful because optical surveys often use point-like morphology as a criterion for selecting AGNs (QSOs) among numerous other objects, and thus are likely to miss moderate-luminosity intermediate-high redshift AGNs embedded in their host galaxies. Also, radio surveys sample only a minor population of AGNs.

The Röntgen satellite ( $R O S A T$ ) provided us with soft $\mathrm{X}$-ray surveys with various depths, ranging from the ROSAT All-Sky Survey (RASS, Voges et al. 1999) to the ROSAT Deep Survey (RDS) on the Lockman Hole (Hasinger et al. 1998). Various optical identification programs of the survey fields have been conducted and the combination of these now enabled us to construct the soft

Send offprint requests to: T. Miyaji, e-mail: miyaji@astro.phys.cmu.edu
X-ray luminosity function (SXLF) as a function of redshift.

In Paper I, we presented a number of global representations of the $0.5-2 \mathrm{keV}$ SXLF and investigated the contribution to the soft X-ray background. We showed that our data are not consistent with the pure-luminosity evolution (PLE), contrary to the suggestions of a number of previous analyses (e.g. Boyle et al. 1994; Jones et al. 1996). Instead, we find an excess of intermediateredshift low-luminosity AGNs above the PLE case, some sign of which was also recognized by Page et al. (1997). In view of this, we developed two versions of luminositydependent density evolution (LDDE1 and LDDE2) models, which represent the observed data very well. An extrapolation of these two LDDE models below the faintest limit of the survey $\left(S_{\mathrm{x}}=1.710^{-15}\left[\mathrm{erg} \mathrm{s}^{-1} \mathrm{~cm}^{-2}\right]\right)$ yields significantly different predictions for fainter fluxes, bracketing the range of a possible AGN contribution to the soft X-ray Background. Chandra (e.g. Mushotzky et al. 2000; Hornschemeier et al. 2000) and XMM-Newton (2001) are probing much fainter sources and spectroscopic identifications of these will eventually show which of these models is closer to the actual behavior of the AGN SXLF. However, because at least some of the faint X-ray sources are optically too faint for spectroscopic identification even 
Table 1. ROSAT surveys used in the analysis

\begin{tabular}{lccc}
\hline \hline Survey $^{\mathrm{a}}$ & $\begin{array}{c}S_{\mathrm{x} 14}^{\mathrm{lim}} \\
10^{-14}\left[\mathrm{erg} \mathrm{s}^{-1} \mathrm{~cm}^{-2}\right]\end{array}$ & $\begin{array}{c}\text { Area } \\
{\left[\mathrm{deg}^{2}\right]}\end{array}$ & $\begin{array}{c}\text { No. of } \\
\text { AGNs }\end{array}$ \\
\hline RBS & $\approx 250$ & $2.010^{4}$ & 216 \\
SA-N & $\approx 13$ & 685. & 130 \\
RIXOS & 3.0 & 15. & 205 \\
NEP & 1.0 & 0.21 & 13 \\
UKD & 0.5 & 0.16 & 29 \\
RDS-Marano & 0.5 & 0.20 & 30 \\
RDS-LH & $0.17-0.9$ & 0.30 & 68 \\
\hline
\end{tabular}

a Abbreviations - RBS: The ROSAT Bright Survey (Fischer et al. 1998; Schwope et al. 2000), SA-N: The Selected AreaNorth (Zickgraf et al. 1997; Appenzeller et al. 1998), RIXOS: The ROSAT International X-ray Optical Survey (Mason et al. 1999), NEP: The North Ecliptic Pole Survey (Bower et al. 1996); UKD: The UK Deep Survey (McHardy et al. 1998), RDS-Marano: The ROSAT Deep Survey - Marano field (Zamorani et al. 1999, RDS-LH: The ROSAT Deep Survey - Lockman Hole (Hasinger et al. 1998; Schmidt et al. 1998; Lehmann et al. 1999). ${ }^{\mathrm{b}}$ Excluding AGNs with $z<0.015$.

with the largest telescopes, extending the XLF into such faint flux level may be difficult.

In this second paper, we present practical and convenient expressions of the observed SXLF from the ROSAT surveys. We present our results mainly for the investigators who are interested in particular redshift regimes and/or comparing their models with observations. For this purpose, we show convenient analytical expressions in several redshift intervals separately. These give more accurate representations of the data in the redshift ranges of interest than those presented in Paper I. We also tabulate the numerical values of the binned SXLF using an improved estimator.

We use a Hubble constant $H_{0}=50 h_{50}$ $\left[\mathrm{km} \mathrm{s}^{-1} \mathrm{Mpc}^{-3}\right]$. The $h_{50}$ dependences are explicitly shown. We calculate the results with common sets of cosmological parameters: $\left(\Omega_{\mathrm{m}}, \Omega_{\Lambda}\right)=(1.0,0.0),(0.3,0.0)$. and $\left(\Omega_{\mathrm{m}}, \Omega_{\Lambda}\right)=(0.3,0.7)$. The symbol "Log" represents the base-10 logarithm.

\section{The summary of the sample}

We have used soft X-ray sources identified with AGNs with redshift information from a combination of ROSAT surveys in various depths/areas from a number of already published and unpublished sources. Detailed description of the definition of the sample, ROSAT countrate-to-flux conversion, and survey area are shown in Paper I. The summary of the samples, which is a duplicate of Table 1. of Paper I with updated references, is shown in Table 1. The details of the nature and completeness of each sample were discussed in Paper I. The limiting flux versus survey area relation were also shown in Paper I.

\section{The SXLF estimation}

As in Paper I, we present the SXLF in the observed 0.5$2 \mathrm{keV}$ band, i.e., in the $0.5(1+z)-2(1+z) \mathrm{keV}$ range in the object's rest frame. This is equivalent to assuming an energy index of 1 . Thus no K-correction was applied for our expressions presented here. The reasons for this choice are explained in detail in Paper I. This choice is particularly important for this paper, which is intended to be used as observational constraints for population-synthesis-type models (e.g. Madau et al. 1994; Comastri et al. 1995; Gilli et al. 1999, 2000; Miyaji et al. 2000) with various spectral assumptions. Because of that, it is more useful to provide quantities in a model-independent form rather than applying a particular version of model-dependent K-corrections. By presenting the data in this manner, one can avoid the difficulty of reverse $\mathrm{K}$-correcting and re-applying new K-corrections when the new results from Chandra and $X M M$ provide better knowledge of the $\mathrm{X}$-ray spectra of the population.

\subsection{Analytical expressions}

First, we find a smooth analytical function for each redshift bin using a Maximum-likelihood fitting. The absolute goodness of the resulting expression can then be tested by one- and two-dimensional Kolgomorov-Smirnov tests (hereafter, 1D-KS and 2D-KS tests respectively; Press et al. 1992; Fasano \& Franceschini 1987). See Paper I for detailed description of these methods. These fittings and tests can be applied to unbinned data sets thus are free from artifacts and biases from binning.

For an analytical expression, we use the smoothed twopower-law formula, as we did in Paper I. Here, we fit the data in narrow redshift bins and thus evolution in each redshift bin is assumed to be a pure density evolution form:

$\frac{\mathrm{d} \Phi\left(L_{\mathrm{x}}, z\right)}{\mathrm{d} \log L_{\mathrm{x}}}=A\left[\left(\frac{L_{\mathrm{x}}}{L_{*}}\right)^{\gamma_{1}}+\left(\frac{L_{\mathrm{x}}}{L_{*}}\right)^{\gamma_{2}}\right]^{-1} \cdot\left(\frac{1+z}{1+z_{\mathrm{c}}}\right)^{p}$

where $z_{\mathrm{c}}$ is the central redshift of the bin. For the highest redshift bin where the "break" is not apparent, we have used a single power-law form by neglecting the first term in the square bracket in Eq. (1).

The luminosity range of the fit is from $\log L_{\mathrm{x}}=41.7$ to the maximum available luminosity in the sample. As shown below and in Paper I, the SXLF below the minimum luminosity has a significant excess above the smooth extrapolation. This excess smoothly connects with the SXLF of the non-AGN population (e.g. Hasinger et al. 1999) and the X-ray emission may be significantly contaminated by non-AGN activities.

The set of parameters which give the best fit for each redshift bin are shown in Table 2 along with the results of the 1D- and 2D- KS tests (see the notes of the table). The parameter errors correspond to a likelihood change of 2.7 (90\% confidence errors). In any case, Eq. (1) gives a statistically satisfactory expression for all redshift bins. 
Table 2. Best-fit parameters for each redshift bin

\begin{tabular}{|c|c|c|c|c|c|c|c|c|}
\hline$z$-range & $z_{\mathrm{c}}$ & $\bar{N}$ & $\overline{A^{\mathrm{a}}}$ & 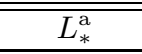 & $\gamma_{1}$ & $\gamma_{2}$ & $p$ & KS-prob $^{b}$ \\
\hline \multicolumn{9}{|c|}{$\left(\Omega_{\mathrm{m}}, \Omega_{\Lambda}\right)=(1.0,0.0)$} \\
\hline $0.015-0.2$ & 0.1 & 269 & $(2.12 \pm 0.23) 10^{-6}$ & $0.60_{-0.32}^{+0.68}$ & $0.59_{-0.29}^{+0.23}$ & $2.1_{-0.3}^{+0.4}$ & $4.22_{-2.61}^{+2.53}$ & $0.99,0.68,0.61$ \\
\hline $0.2-0.4$ & 0.3 & 113 & $(3.72 \pm 0.63) 10^{-6}$ & $0.89_{-0.46}^{+1.20}$ & $0.67_{-0.38}^{+0.30}$ & $2.5_{-0.3}^{+0.4}$ & $5.25_{-3.51}^{+3.48}$ & $0.93,0.56,0.72$ \\
\hline $0.4-0.8$ & 0.6 & 99 & $(1.55 \pm 0.28) 10^{-5}$ & $0.54_{-0.29}^{+0.46}$ & $0.33_{-0.87}^{+0.52}$ & $2.2_{-0.2}^{+0.3}$ & $5.95_{-2.29}^{+2.29}$ & $0.85,0.85,0.57$ \\
\hline $0.8-1.6$ & 1.2 & 135 & $(1.75 \pm 0.27) 10^{-5}$ & $1.48_{-0.56}^{+1.14}$ & $0.40_{-0.53}^{+0.41}$ & $2.4_{-0.2}^{+0.2}$ & $4.07_{-1.34}^{+1.33}$ & $0.99,0.96,0.83$ \\
\hline $1.6-2.3$ & 2.2 & 44 & $(3.75 \pm 1.02) 10^{-5}$ & $1.2(*)$ & $0.0(*)$ & $2.1_{-0.1}^{+0.2}$ & $0(*)$ & $0.27,0.36,0.19$ \\
\hline $2.3-4.6$ & 3.0 & 25 & $(3.93 \pm 1.41) 10^{-5}$ & $1.0(*)$ & $\ldots$ & $1.9_{-0.2}^{+0.2}$ & $0(*)$ & $0.72,0.99,0.64$ \\
\hline \multicolumn{9}{|c|}{$\left(\Omega_{\mathrm{m}}, \Omega_{\Lambda}\right)=(0.3,0.0)$} \\
\hline $0.015-0.2$ & 0.1 & 269 & 3) $10^{-6}$ & $0.59_{-0.32}^{+0.71}$ & $0.59_{-0.30}^{+0.23}$ & & & $0.99,0.6$ \\
\hline $0.2-0.4$ & 0.3 & 113 & $(3.33 \pm 0.56) 10^{-6}$ & $0.93_{-0.49}^{+1.30}$ & $0.67_{-0.39}^{+0.31}$ & $2.4_{-0.3}^{+0.4}$ & $5.31_{-3.51}^{+3.49}$ & $0.99,0.53,0.79$ \\
\hline $0.4-0.8$ & 0.6 & 99 & $(1.03 \pm 0.19) 10^{-5}$ & $0.69_{-0.36}^{+1.07}$ & $0.37_{-0.80}^{+0.50}$ & $2.3_{-0.2}^{+0.3}$ & $5.90_{-2.28}^{+2.28}$ & $0.97,0.81,0.57$ \\
\hline $0.8-1.6$ & 1.2 & 135 & $(9.28 \pm 1.44) 10^{-6}$ & $2.14_{-0.83}^{+1.67}$ & $0.42_{-0.52}^{+0.40}$ & $2.4_{-0.2}^{+0.2}$ & $4.13_{-1.34}^{+1.38}$ & $0.98,0.97,0.72$ \\
\hline $1.6-2.3$ & 2.2 & 44 & $(1.90 \pm 0.51) 10^{-5}$ & $1.8(*)$ & $0.0\left(^{*}\right)$ & $2.1_{-0.1}^{+0.2}$ & $0(*)$ & $0.19,0.41,0.15$ \\
\hline $2.3-4.6$ & 3.0 & 25 & $(5.00 \pm 1.80) 10^{-5}$ & $1.0\left(^{*}\right)$ & $\ldots$ & $1.9_{-0.2}^{+0.2}$ & $0(*)$ & $0.64,0.96,0.75$ \\
\hline \multicolumn{9}{|c|}{$\left(\Omega_{\mathrm{m}}, \Omega_{\Lambda}\right)=(0.3,0.7)$} \\
\hline $0.015-0.2$ & 0.1 & 269 & $(1.58 \pm 0.17) 10^{-6}$ & $0.71_{-0.39}^{+0.86}$ & $0.62_{-0.29}^{+0.22}$ & $2.1_{-0.3}^{+0.4}$ & $3.79_{-2.64}^{+2.56}$ & $0.99,0.54,0.61$ \\
\hline $0.2-0.4$ & 0.3 & 113 & $(2.40 \pm 0.41) 10^{-6}$ & $1.09_{-0.58}^{+1.53}$ & $0.67_{-0.39}^{+0.30}$ & $2.4_{-0.3}^{+0.3}$ & $4.95_{-3.51}^{+3.49}$ & $0.97,0.56,0.82$ \\
\hline $0.4-0.8$ & 0.6 & 99 & $(6.71 \pm 1.22) 10^{-6}$ & $0.85_{-0.46}^{+1.41}$ & $0.36_{-0.87}^{+0.51}$ & $2.2_{-0.2}^{+0.3}$ & $5.69_{-2.27}^{+2.28}$ & $0.96,0.81,0.60$ \\
\hline $0.8-1.6$ & 1.2 & 135 & $(5.68 \pm 0.88) 10^{-6}$ & $2.69_{-1.04}^{+2.07}$ & $0.43_{-0.51}^{+0.39}$ & $2.4_{-0.2}^{+0.2}$ & $4.10_{-1.34}^{+1.33}$ & $0.98,0.97,0.74$ \\
\hline $1.6-2.3$ & 2.2 & 44 & $(1.34 \pm 0.36) 10^{-5}$ & $2.0(*)$ & $0.0(*)$ & $2.1_{-0.1}^{+0.1}$ & $0(*)$ & $0.14,0.40,0.16$ \\
\hline $2.3-4.6$ & 3.0 & 25 & $(4.25 \pm 1.53) 10^{-5}$ & $1.0(*)$ & $\ldots$ & $1.9_{-0.2}^{+0.2}$ & $0\left(^{*}\right)$ & $0.78,0.98,0.76$ \\
\hline
\end{tabular}

Parameter values which have been fixed during the fit are labeled by "(*)". ${ }^{\mathrm{a}} \mathrm{Units}-\mathrm{A}:\left[h_{50}^{3} \mathrm{Mpc}^{-3}\right], L_{*}$ : $\left[10^{44} h_{50}^{-2} \mathrm{erg} \mathrm{s}^{-1}\right]{ }^{\mathrm{b}}$ The three values are probabilities in two $1 \mathrm{D}-\mathrm{KS}$ test for the distribution, $L_{\mathrm{x}}, 1 \mathrm{D}-\mathrm{KS}$ test for the $z$ distribution and the 2D-KS test for the $\left(L_{\mathrm{x}}, z\right)$ space respectively.

\subsection{The $\sum V_{a}^{-1}$ method}

The $\sum V_{\mathrm{a}}^{-1}$ estimator, which is a generalized version of the original $\sum V_{\max }^{-1}$ estimator (Schmidt 1968) applied to a sample composed of subsamples of different depths (see Paper I; Avni \& Bahcall 1980), has been widely used for binned luminosity functions (LF; we use the acronym LF when the discussion is not limited to the luminosity function in the X-ray band) in the literature. However, as discussed in Paper I (see also Wisotzki 1998; Page \& Carrera 1999), using it for a binned LF estimator can cause significant biases, especially if the bin covers the flux range where the available solid angle of the survey changes rapidly as a function of flux. Also, the choice of the location in a $\log L_{\mathrm{x}}$ bin with a non-negligible width at which the data point is plotted significantly changes the impression of the plot. In Fig. 3 of Paper I, however, we plotted the $\sum V_{\mathrm{a}}^{-1}$ estimates, because of the lack of a reasonable alternative at the time of writing that paper, with caveats on biases associated with the method. We note that the estimator can be used in an unbinned manner by considering a set of delta-functions weighted by $V_{\mathrm{a}}^{-1}$ (or $V_{\max }^{-1}$ ) at the positions of sample objects in the luminosity space (Schmidt \& Green 1983), and this method is free from biases mentioned above. While this unbinned method is a powerful tool to predict, e.g., the source counts, it does not provide practical means of plotting. In this paper, we have developed an improved estimator, which is explained in the next subsection.

\subsection{An improved estimator of the binned SXLF}

As an alternative to the $\sum V_{\mathrm{a}}^{-1}$ method, we have developed the following estimator for the binned LF, which is free from most biases unavoidable in the $\sum V_{\mathrm{a}}^{-1}$ method. In Sect. 3.1, we have found a smooth analytical function which describes the behavior of the SXLF in a given redshift range. Having the best-fit smooth function, the estimated numerical value for the SXLF in a given bin in the $\left(L_{\mathrm{x}}, z\right)$-space is:

$$
\frac{\mathrm{d} \Phi^{\mathrm{n}}}{\mathrm{d} \log L_{\mathrm{x}}}\left(L_{\mathrm{x} i}, z_{i}\right)=\frac{\mathrm{d} \Phi^{\mathrm{mdl}}}{\mathrm{d} \log L_{\mathrm{x}}}\left(L_{\mathrm{x} i}, z_{i}\right) \cdot \frac{N_{i}^{\mathrm{obs}}}{N_{i}^{\mathrm{mdl}}},
$$

where $L_{\mathrm{x} i}$ and $z_{i}$ are the luminosity and redshift representative of the $i$ th $\operatorname{bin}, \frac{\mathrm{d} \Phi^{\mathrm{mdl}}}{\mathrm{d} \log L_{\mathrm{x}}}$ is the best-fit analytical expression evaluated at this point, $N_{i}^{\text {obs }}$ is the actual number of AGNs observed in the $i$ th bin, and $N_{i}^{\text {mdl }}$ is the predicted number of AGNs in the bin from the best-fit analytical expression. Hereafter, we refer to Eq. (2) as the " $N^{\text {obs }} / N^{\text {mdl }}$ estimator". Note that the estimator proposed by Page \& Carrera (1999) (hereafter PC) is a special case of Eq. (2) where $\frac{\mathrm{d} \Phi^{\mathrm{mdl}}}{\mathrm{d} L_{\mathrm{x}}} \propto$ const (or $\frac{\mathrm{d} \Phi^{\mathrm{mdl}}}{\mathrm{d} \log L_{\mathrm{x}}} \propto L_{\mathrm{x}}$ ).

Another advantage of this estimator over $\sim V_{\mathrm{a}}^{-1}$ is that exact errors at a given significance can be evaluated using Poisson statistics. One disadvantage of this estimator is that it is model-dependent, at least in principle. Since our analytical expressions are satisfactory representations in any case and the estimator is not sensitive to the details of 


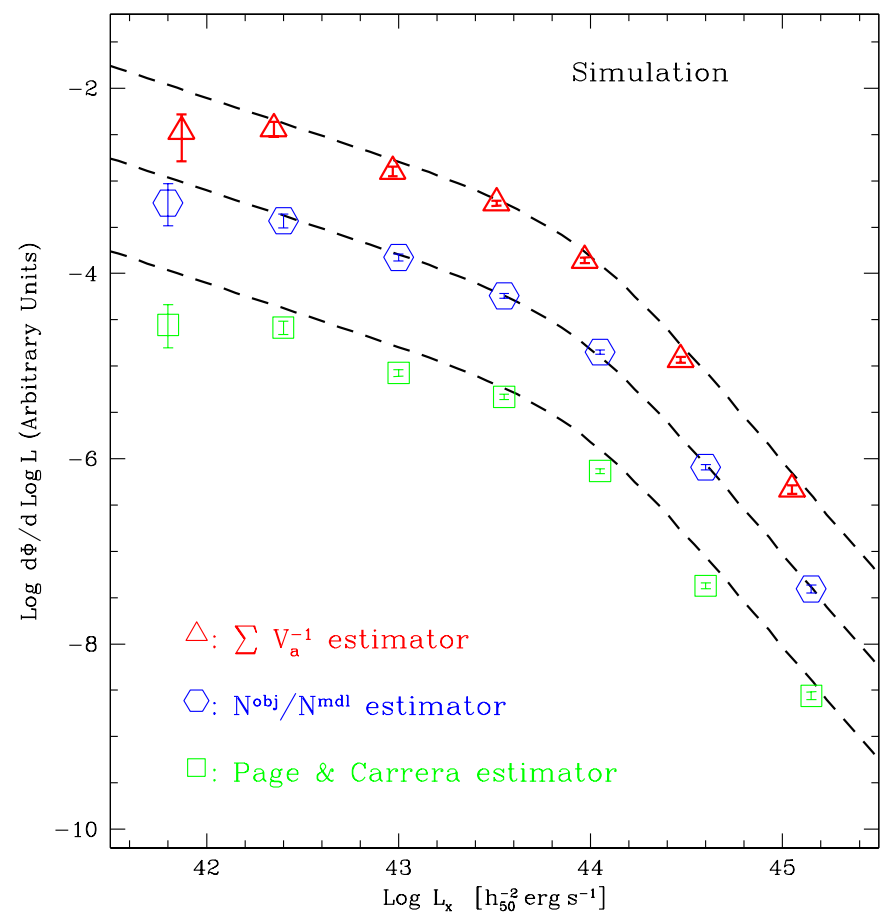

Fig. 1. The binned XLFs from a simulated sample using three different estimators (symbols with error bars as labeled) are compared with the underlying "true" XLF represented by dashed lines. The vertical positions of the three different estimators have been shifted vertically for display

the underlying model, the uncertainties due to the model dependence are practically negligible.

In order to compare the goodness of the estimators, we performed simulations. Using the actual best-fit model for the $0.2<z<0.4$ bin for $\left(\Omega_{\mathrm{m}}, \Omega_{\Lambda}\right)=(1.0,0.0)$, we generated a set of simulated AGNs. The number of simulated AGNs are 10 times those of the actual sample in order to reduce the Poisson errors. Using the simulated AGNs and the actual flux-area relation of our combined sample, we estimated binned SXLFs using three different estimators: $\sum V_{\mathrm{a}}^{-1}, N^{\mathrm{obs}} / N^{\mathrm{mdl}}$ (Eq. (2)) and that of PC. The results are compared with the underlying SXLF, which was used to generate the simulated AGNs, in Fig. 1. For the models to evaluate $N^{\mathrm{mdl}}$, we used the re-fitted model using the simulated sample rather than the original model. The $1 \sigma$ errors for the $N^{\text {obs }} / N^{\text {mdl }}$ (Eq. (2)) and the PC estimators are Poisson errors calculated using Eqs. (7) and (12) of Gehrels (1986). On the other hand, the errors for the $\sum V_{\mathrm{a}}^{-1}$ estimator are from Eq. (3) of Paper I and are inaccurate for bins with a small number of AGNs.

As shown in Fig. 1, the $N^{\text {obs }} / N^{\text {mdl }}$ estimator best represents the original model and no estimated point deviates from the underlying model by more than $2 \sigma$. The $\sum V_{\mathrm{a}}^{-1}$ estimator underestimates the XLF in the lowest luminosity bin as found in PC. We note that the PC estimator also systematically underestimates the LF in this particular case of the underlying model and the flux-area relation. This is expected because their estimator implicitly builds in the assumption $\frac{\mathrm{d}^{\mathrm{mdl}}}{\mathrm{dL}_{\mathrm{x}}} \propto$ const as the underlying $\mathrm{LF}$ shape. This is much more weighted towards higher luminosities than any part of the realistic AGN XLF. Since the amount of this bias depends on the underlying model and the flux-area relation, as well as the points in the bin where the data are plotted, it is not surprising that the bias is not apparent in Fig. 2 of PC. They have also compensated for this bias upon comparing the estimated LF with a model. Instead of correcting the estimated binned LF using a good model (which our $N^{\text {obs }} / N^{\text {mdl }}$ estimator does), they calculated the "model-expectated value of the estimator" to compare with the estimated value from the data. Detailed investigation and comparison of these different estimators in various cases are beyond the scope of this paper. Judging from this simulation, the above discussion on biases, and that the exact Poisson errors can be used for errors, we choose to use the $N^{\text {obs }} / N^{\text {mdl }}$ estimator for our plots and tabulation.

\subsection{The binned SXLF results}

Using the $N^{\text {obs }} / N^{\text {mdl }}$ estimator, we revised the full SXLF plot (Fig. 3 of Paper I), as shown in Fig. 2. Instead of connecting the data points, we overplotted the analytical model for each redshift bin.

The resulting binned SXLF are listed in Tables 3, 4, and 5 for different sets of cosmological parameters respectively. The columns of these tables are - (1) the redshift range of the bin; (2) the luminosity range of the bin; (3) the number of AGNs in the sample for the bin and the number of non- "type 1" AGNs as defined in Appendix A of Paper I; (4) the number of AGNs expected from the analytical model derived in Sect. 3.1. (5) the binned SXLF estimated using Eq. (2) using the model XLF evaluated at the central point of the bin, i.e. $z=z_{\mathrm{c}}$ (see Table 2) and $\log L_{\mathrm{x}}=\left(\log L_{\mathrm{xmin}}+\log L_{\mathrm{xmax}}\right) / 2$, where the subscripts min and max signify the borders of the bin in Cols. (1) and (2). The upper and lower errors correspond to Poisson errors estimated by Eqs. (7) and (12) of Gehrels (1986) respectively using $S=1$ (corresponding to the confidence of the Gaussian $1 \sigma$. When there is no object in the bin, the Poissonian 90\% confidence upper-limit is given (corresponding to 2.3 objects). We recommend use of the values and errors under this column when, e.g. overplotting observed SXLF values with model predictions ${ }^{1}$.

Figure 2 shows that the lowest-redshift, lowestluminosity bin has a significant excess over the two powerlaw analytical expression (AGNs belonging to this bin have not been used for the two power-law fit), thus the actual underlying SXLF has a much steeper slope than that used for $N^{\text {mdl }}$. In order to evaluate the bias caused by this, we have made an $N^{\text {obs }} / N^{\text {mdl }}$ estimate of this particular bin using the local slope of $\gamma=1.7$ instead of $\gamma \sim 0.6$ from the two power-law model. This gave a value about

${ }^{1}$ The ASCII versions of these tables with additional columns and separate tables for the "type I" sample are provided as a part of the source distribution in the preprint archive (http://xxx.lanl.gov/, astro-ph/0101279). 


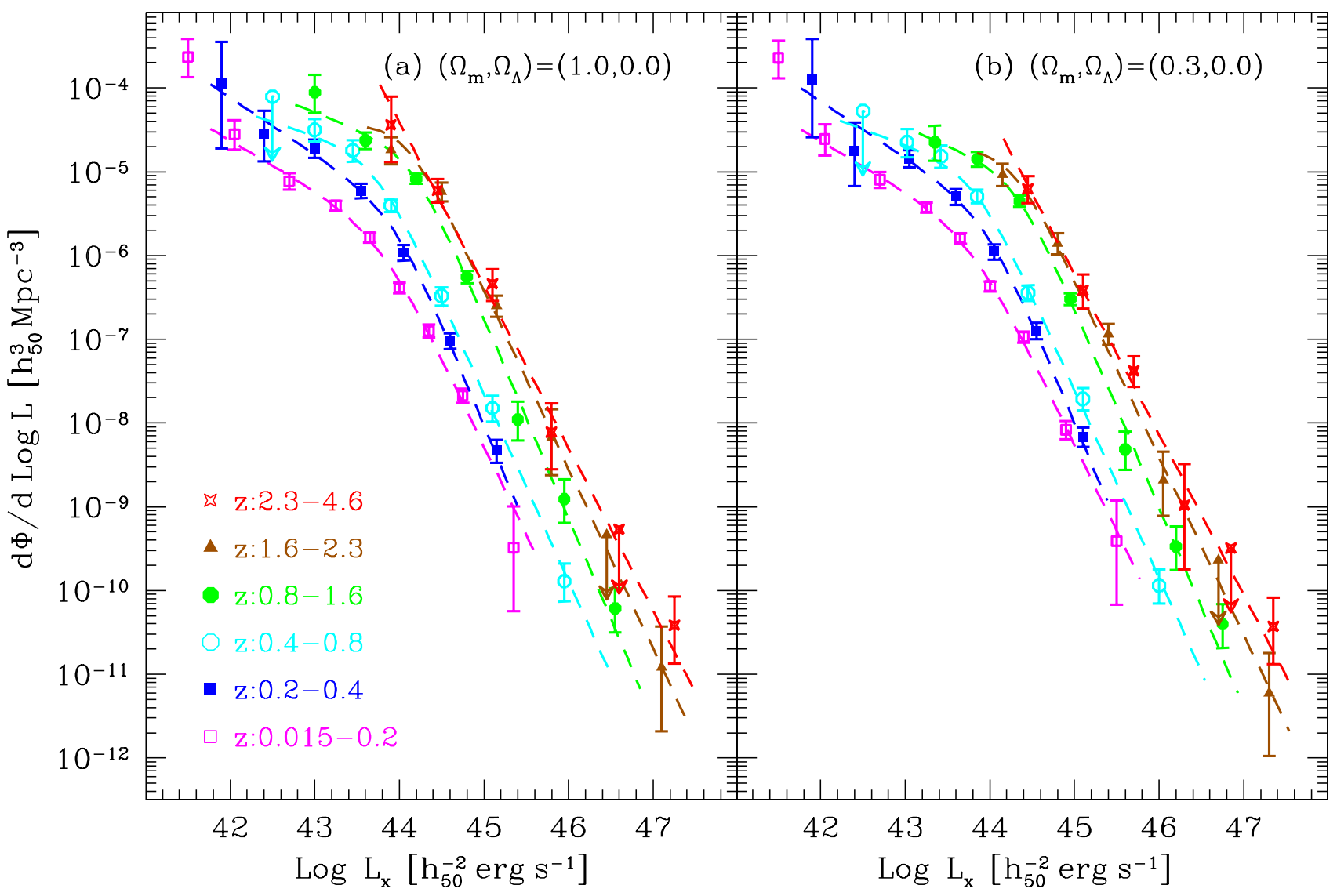

Fig. 2. The binned SXLFs estimated by Eq. (2) are plotted with Poisson errors corresponding to the significance range of Gaussian $1 \sigma$ from Eqs. (7) and (12) by Gehrels (1986). The data points and error estimates are more accurate than Fig. 3 of Paper I. Different symbols correspond to different redshift bins as indicated in the lower-left part of panel a). The symbol attached to a downward arrow indicates the $90 \%$ upper limit (corresponding to 2.3 objects) for the bin with no AGN in the sample. The best-fit analytical model for each redshift bin in the luminosity range used for the fit is overplotted in dashed lines

$10 \%$ lower, thus, the difference is much smaller than the statistical errors for this bin.

\section{Discussion}

We have shown the tables of observed SXLF values for a number of standard set of cosmological parameters. These values are intended for direct comparison with models and plotting with realistic error bars. However, we list a number of caveats and sources of uncertainties, and related issues.

- Countrate-to-flux conversion: For the PSPC-based observations, where we can limit pulse-height channels, the uncertainty in the countrate-flux (in $0.5-2[\mathrm{keV}]$ ) conversion is small $( \pm 3 \%$ for photon spectral index of $\Gamma=2.0 \pm 0.7$. At the faintest end $\left(S_{\mathrm{x} 14}<0.5\right)$, where only the HRI data are available, the conversion rate varies by $\pm 40 \%$ for the same spectral index range.

- Optical classification of AGNs: Since different catalogs used in this analysis have different criteria for type I and type II AGNs, we did not show separate expressions for these two populations. See Appendix A. of Paper I for the approximate difference in behavior of the tentative "type I" sample.
- Incompleteness: Most of the surveys used in the analysis are highly complete or we have selected an appropriate complete subset. In case there is incompleteness, we have corrected for it by assuming that the redshift distribution/content of the remaining sources are the same as the identified ones in the same flux range. This assumption is not likely to be the case, considering that they have not been identified not because of a random cause but because of optical faintness and difficulty in obtaining decent optical spectra. The only place that this could affect significantly is the faintest end of RDS-LH $\left(0.17 \leq S_{\mathrm{x} 14} \leq 0.38\right)$, where the identification completeness is $\sim 80 \%$. (At $S_{\mathrm{x} 14} \geq 0.38$, the identification completeness is $\gtrsim 95 \%$ in any flux range). This could affect the behavior of, e.g. the apparent break at the low luminosity end in $1.6 \leq z<2.3$.

- X-ray spectra/absorption: A serious model composer should be aware that the luminosity given here is for $0.5-2[\mathrm{keV}]$ in the observer frame. Thus, one should compare the model, with their own spectral assumptions (spectral index, absorption, fraction of absorbed AGNs which may depend on luminosity/redshift), should compute the apparent luminosity in the $0.5(1+$ $z)-2(1+z)[\mathrm{keV}]$ range and compare it with the 
Table 3. The full binned SXLF values -1

\begin{tabular}{|c|c|c|c|c|}
\hline \multicolumn{5}{|c|}{ 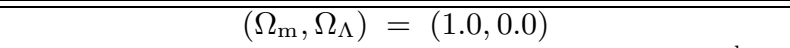 } \\
\hline $\begin{array}{c}z \\
(1) \\
\end{array}$ & $\begin{array}{c}\log L_{\mathrm{x}}{ }^{\mathrm{a}} \\
(2)\end{array}$ & $\begin{array}{l}N^{\text {obs }} \\
(3) \\
\end{array}$ & $\begin{array}{c}N^{\text {mdl }} \\
(4) \\
\end{array}$ & $\begin{array}{c}\frac{\mathrm{d} \Phi^{\mathrm{n}}}{\mathrm{d} \log L_{\mathrm{x}}} \\
(5)\end{array}$ \\
\hline $.015-0.2$ & $41.30-41.70$ & $5(3)$ & 1.0 & $\left(2.3_{-1.0}^{+1.5}\right) 10^{-4}$ \\
\hline $.015-0.2$ & $41.70-42.40$ & $8(2)$ & 6.3 & $\left(2.8_{-1.0}^{+1.3}\right) 10^{-5}$ \\
\hline $.015-0.2$ & $42.40-43.00$ & $23(3)$ & 26.8 & $\left(7.7_{-1.6}^{+1.9}\right) 10^{-6}$ \\
\hline $.015-0.2$ & $43.00-43.50$ & $61(7)$ & 58.4 & $\left(3.9_{-0.5}^{+0.0}\right) 10^{-6}$ \\
\hline $.015-0.2$ & $43.50-43.80$ & $56(3)$ & 52.6 & $\left(1.6_{-0.2}^{+0.2}\right) 10^{-6}$ \\
\hline $.015-0.2$ & $43.80-44.20$ & $55(4)$ & 65.5 & $\left(4.1_{-0.6}^{+0.6}\right) 10^{-7}$ \\
\hline $.015-0.2$ & $44.20-44.50$ & $35(1)$ & 31.6 & $\left(1.3_{-0.2}^{+0.2}\right) 10^{-7}$ \\
\hline $.015-0.2$ & $44.50-45.00$ & 30 & 25.0 & $\left(2.1_{-0.4}^{+0.4}\right) 10^{-8}$ \\
\hline $.015-0.2$ & $45.00-45.70$ & 1 & 3.0 & $\left(3.3_{-2.7}^{+6.8}\right) 10^{-10}$ \\
\hline $0.2-0.4$ & $41.70-42.10$ & 1 & 0.8 & $\left(1.1_{-0.9}^{+2.3}\right) 10^{-4}$ \\
\hline $0.2-0.4$ & $42.10-42.70$ & $3(2)$ & 4.3 & $\left(2.8_{-15}^{+2.5}\right) 10^{-5}$ \\
\hline $0.2-0.4$ & $42.70-43.30$ & $18(5)$ & 15.1 & $\left(1.9_{-0.4}^{+0.5}\right) 10^{-5}$ \\
\hline $0.2-0.4$ & $43.30-43.80$ & $27(3)$ & 26.3 & $\left(5.9_{-1.1}^{+1.3}\right) 10^{-6}$ \\
\hline $0.2-0.4$ & $43.80-44.30$ & $25(3)$ & 29.0 & $\left(1.1_{-0.2}^{+0.3}\right) 10^{-6}$ \\
\hline $0.2-0.4$ & $44.30-44.90$ & $26(1)$ & 23.5 & $\left(9.6_{-1.9}^{+2.2}\right) 10^{-8}$ \\
\hline $0.2-0.4$ & $44.90-45.40$ & 13 & 11.3 & $\left(4.7_{-1.3}^{+1.6}\right) 10^{-9}$ \\
\hline $0.4-0.8$ & $42.30-42.70$ & 0 & 1.1 & $<7.910^{-5}$ \\
\hline $0.4-0.8$ & $42.70-43.30$ & $13(7)$ & 10.8 & $\left(3.1_{-0.9}^{+1.1}\right) 10^{-5}$ \\
\hline $0.4-0.8$ & $43.30-43.60$ & $14(4)$ & 11.7 & $\left(1.8_{-0.5}^{+0.6}\right) 10^{-5}$ \\
\hline $0.4-0.8$ & $43.60-44.20$ & 38 & 43.2 & $\left(3.9_{-0.6}^{+0.5}\right) 10^{-6}$ \\
\hline $0.4-0.8$ & $44.20-44.80$ & $19(1)$ & 16.8 & $\left(3.3_{-0.7}^{+0.9}\right) 10^{-7}$ \\
\hline $0.4-0.8$ & $44.80-45.40$ & 10 & 8.9 & $\left(1.5_{-0.5}^{+0.6}\right) 10^{-8}$ \\
\hline $0.4-0.8$ & $45.40-46.50$ & 5 & 6.5 & $\left(1.3_{-0.6}^{+0.8}\right) 10^{-10}$ \\
\hline $0.8-1.6$ & $42.70-43.30$ & 5 & 2.9 & $\left(8.9_{-3.8}^{+5.6}\right) 10^{-5}$ \\
\hline $0.8-1.6$ & $43.30-43.90$ & $23(2)$ & 26.9 & $\left(2.4_{-0.5}^{+0.6}\right) 10^{-5}$ \\
\hline $0.8-1.6$ & $43.90-44.50$ & 55 & 53.0 & $\left(8.2_{-1.1}^{+1.2}\right) 10^{-6}$ \\
\hline $0.8-1.6$ & $44.50-45.10$ & 39 & 36.0 & $\left(5.6_{-0.9}^{+1.0}\right) 10^{-7}$ \\
\hline $0.8-1.6$ & $45.10-45.70$ & 5 & 9.0 & $\left(1.1_{-0.5}^{+0.7}\right) 10^{-8}$ \\
\hline $0.8-1.6$ & $45.70-46.20$ & 4 & 3.1 & $\left(1.2_{-0.6}^{+0.9}\right) 10^{-9}$ \\
\hline $0.8-1.6$ & $46.20-46.90$ & 4 & 2.3 & $\left(6.1_{-2.9}^{+4.5}\right) 10^{-11}$ \\
\hline $1.6-2.3$ & $43.60-44.20$ & 9 & 13.1 & $\left(1.8_{-0.6}^{+0.8}\right) 10^{-5}$ \\
\hline $1.6-2.3$ & $44.20-44.80$ & 18 & 12.9 & $\left(5.8_{-1.4}^{+1.6}\right) 10^{-6}$ \\
\hline $1.6-2.3$ & $44.80-45.50$ & 14 & 10.5 & $\left(2.5_{-0.7}^{+0.8}\right) 10^{-7}$ \\
\hline $1.6-2.3$ & $45.50-46.10$ & 2 & 2.3 & $\left(6.7_{-4.3}^{+8.0}\right) 10^{-9}$ \\
\hline $1.6-2.3$ & $46.10-46.80$ & 0 & 1.5 & $<4.610^{-10}$ \\
\hline $1.6-2.3$ & $46.80-47.40$ & 1 & 1.0 & $\left(1.2_{-1.0}^{+2.5}\right) 10^{-11}$ \\
\hline $2.3-4.6$ & $43.70-44.10$ & 2 & 3.4 & $\left(3.6_{-2.3}^{+4.3}\right) 10^{-5}$ \\
\hline $2.3-4.6$ & $44.10-44.80$ & 12 & 10.5 & $\left(6.0_{-1.7}^{+2.2}\right) 10^{-6}$ \\
\hline $2.3-4.6$ & $44.80-45.40$ & 7 & 4.3 & $\left(4.6_{-1.7}^{+2.3}\right) 10^{-7}$ \\
\hline $2.3-4.6$ & $45.40-46.20$ & 2 & 3.2 & $\left(7.8_{-5.0}^{+9.4}\right) 10^{-9}$ \\
\hline $2.3-4.6$ & $46.20-47.00$ & 0 & 1.4 & $<5.410^{-10}$ \\
\hline $2.3-4.6$ & $47.00-47.50$ & 2 & 1.0 & $\left(3.8_{-2.5}^{+4.6}\right) 10^{-11}$ \\
\hline
\end{tabular}

Notes: ${ }^{\mathrm{a}} L_{\mathrm{x}}\left[h_{50}^{-2} \mathrm{erg} \mathrm{s}^{-1}\right]$ in $0.5-2[\mathrm{keV}] .{ }^{\mathrm{b}}\left[h_{50}^{3} \mathrm{Mpc}^{-3}\right]$. The AGNs in the first row are outside of the luminosity range used for the model fit. The numbers of non-type I AGNs are enclosed by parenthesis in Col. (3).

values listed in this paper. The latest population synthesis model based on absorbed and unabsorbed
Table 4. The full binned SXLF values -2

\begin{tabular}{|c|c|c|c|c|}
\hline \multicolumn{5}{|c|}{$\left(\Omega_{\mathrm{m}}, \Omega_{\Lambda}\right)=(0.3,0.0)$} \\
\hline $\begin{array}{c}z \\
(1)\end{array}$ & $\begin{array}{c}\log L_{\mathrm{x}}{ }^{\mathrm{a}} \\
(2)\end{array}$ & $\begin{array}{c}N^{\text {obs }} \\
(3)\end{array}$ & $\begin{array}{c}N^{\mathrm{mdl}} \\
(4)\end{array}$ & $\frac{\mathrm{d} \Phi^{\mathrm{n}}}{\mathrm{d} \log L_{\mathrm{x}}} \mathrm{b}$ \\
\hline $.015-0.2$ & $41.30-41.70$ & $5(3)$ & 1.0 & $\left(2.3_{-1.0}^{+1.4}\right) 10^{-4}$ \\
\hline $.015-0.2$ & $41.70-42.40$ & $7(2)$ & 6.1 & $\left(2.5_{-0.9}^{+1.2}\right) 10^{-5}$ \\
\hline $.015-0.2$ & $42.40-43.00$ & $24(3)$ & 26.0 & $\left(8.1_{-1.6}^{+1.9}\right) 10^{-6}$ \\
\hline $.015-0.2$ & $43.00-43.50$ & $58(6)$ & 56.4 & $\left(3.7_{-0.5}^{+0.5}\right) 10^{-6}$ \\
\hline $.015-0.2$ & $43.50-43.80$ & $55(4)$ & 50.5 & $\left(1.6_{-0.2}^{+0.2}\right) 10^{-6}$ \\
\hline $.015-0.2$ & $43.80-44.20$ & $58(4)$ & 64.4 & $\left(4.3_{-0.6}^{+0.6}\right) 10^{-7}$ \\
\hline $.015-0.2$ & $44.20-44.60$ & $47(1)$ & 39.8 & $\left(1.1_{-0.2}^{+0.2}\right) 10^{-7}$ \\
\hline $.015-0.2$ & $44.60-45.20$ & 19 & 20.6 & $\left(8.2_{-1.9}^{+2.3}\right) 10^{-9}$ \\
\hline $.015-0.2$ & $45.20-45.80$ & 1 & 1.3 & $\left(3.9_{-3.2}^{+8.0}\right) 10^{-10}$ \\
\hline $0.2-0.4$ & $41.70-42.10$ & 1 & 0.6 & $\left(1.3_{-1.0}^{+2.6}\right) 10^{-4}$ \\
\hline $0.2-0.4$ & $42.10-42.70$ & $2(1)$ & 4.2 & $\left(1.8_{-1.1}^{+2.1}\right) 10^{-5}$ \\
\hline $0.2-0.4$ & $42.70-43.40$ & $21(6)$ & 19.4 & $\left(1.4_{-0.3}^{+0.4}\right) 10^{-5}$ \\
\hline $0.2-0.4$ & $43.40-43.80$ & $23(3)$ & 22.0 & $\left(5.0_{-1.0}^{+1.2}\right) 10^{-6}$ \\
\hline $0.2-0.4$ & $43.80-44.30$ & $26(3)$ & 28.7 & $\left(1.1_{-0.2}^{+0.3}\right) 10^{-6}$ \\
\hline $0.2-0.4$ & $44.30-44.80$ & $23(1)$ & 21.3 & $\left(1.3_{-0.3}^{+0.3}\right) 10^{-7}$ \\
\hline $0.2-0.4$ & $44.80-45.40$ & 17 & 14.6 & $\left(6.8_{-1.6}^{+2.0}\right) 10^{-9}$ \\
\hline $0.4-0.8$ & $42.20-42.80$ & 0 & 1.4 & $<5.310^{-5}$ \\
\hline $0.4-0.8$ & $42.80-43.25$ & $8(5)$ & 7.1 & $\left(2.3_{-0.8}^{+1.0}\right) 10^{-5}$ \\
\hline $0.4-0.8$ & $43.25-43.60$ & $13(6)$ & 10.7 & $\left(1.5_{-0.4}^{+0.5}\right) 10^{-5}$ \\
\hline $0.4-0.8$ & $43.60-44.10$ & 34 & 33.4 & $\left(5.1_{-0.9}^{+1.0}\right) 10^{-6}$ \\
\hline $0.4-0.8$ & $44.10-44.80$ & 25 & 28.4 & $\left(3.6_{-0.7}^{+0.8}\right) 10^{-7}$ \\
\hline $0.4-0.8$ & $44.80-45.40$ & $13(1)$ & 10.0 & $\left(1.9_{-0.5}^{+0.7}\right) 10^{-8}$ \\
\hline $0.4-0.8$ & $45.40-46.60$ & 6 & 7.4 & $\left(1.1_{-0.5}^{+0.6}\right) 10^{-10}$ \\
\hline $0.8-1.6$ & $43.10-43.60$ & 6 & 6.3 & $\left(2.2_{-0.9}^{+1.3}\right) 10^{-5}$ \\
\hline $0.8-1.6$ & $43.60-44.10$ & $28(2)$ & 26.1 & $\left(1.4_{-0.3}^{+0.3}\right) 10^{-5}$ \\
\hline $0.8-1.6$ & $44.10-44.60$ & 46 & 45.0 & $\left(4.5_{-0.7}^{+0.7}\right) 10^{-6}$ \\
\hline $0.8-1.6$ & $44.60-45.30$ & 42 & 40.6 & $\left(3.0_{-0.5}^{+0.5}\right) 10^{-7}$ \\
\hline $0.8-1.6$ & $45.30-45.90$ & 5 & 9.0 & $\left(4.8_{-2.1}^{+3.0}\right) 10^{-9}$ \\
\hline $0.8-1.6$ & $45.90-46.50$ & 4 & 3.9 & $\left(3.4_{-1.6}^{+2.5}\right) 10^{-10}$ \\
\hline $0.8-1.6$ & $46.50-47.00$ & 4 & 1.6 & $\left(4.0_{-1.9}^{+2.9}\right) 10^{-11}$ \\
\hline $1.6-2.3$ & $43.80-44.50$ & 13 & 16.7 & $\left(9.3_{-2.5}^{+3.2}\right) 10^{-6}$ \\
\hline $1.6-2.3$ & $44.50-45.10$ & 14 & 12.5 & $\left(1.4_{-0.4}^{+0.5}\right) 10^{-6}$ \\
\hline $1.6-2.3$ & $45.10-45.70$ & 14 & 8.8 & $\left(1.1_{-0.3}^{+0.4}\right) 10^{-7}$ \\
\hline $1.6-2.3$ & $45.70-46.40$ & 2 & 2.9 & $\left(2.1_{-1.3}^{+2.5}\right) 10^{-9}$ \\
\hline $1.6-2.3$ & $46.40-47.00$ & 0 & 1.3 & $<2.310^{-10}$ \\
\hline $1.6-2.3$ & $47.00-47.60$ & 1 & 1.1 & $\left(6.0_{-4.9}^{+12}\right) 10^{-12}$ \\
\hline $2.3-4.6$ & $44.10-44.80$ & 9 & 9.9 & $\left(6.2_{-2.0}^{+2.7}\right) 10^{-6}$ \\
\hline $2.3-4.6$ & $44.80-45.40$ & 6 & 6.1 & $\left(3.8_{-1.5}^{+2.1}\right) 10^{-7}$ \\
\hline $2.3-4.6$ & $45.40-46.00$ & 7 & 4.6 & $\left(4.2_{-1.5}^{+2.1}\right) 10^{-8}$ \\
\hline $2.3-4.6$ & $46.00-46.60$ & 1 & 1.9 & $\left(1.0_{-0.9}^{+2.2}\right) 10^{-9}$ \\
\hline $2.3-4.6$ & $46.60-47.10$ & 0 & 1.2 & $<3.210^{-10}$ \\
\hline $2.3-4.6$ & $47.10-47.60$ & 2 & 1.0 & $\left(3.7_{-2.4}^{+4.5}\right) 10^{-11}$ \\
\hline
\end{tabular}

See notes to Table 3.

AGNs by Gilli et al. (2000) has applied this approach using the tables shown in this paper. However, as discussed in Paper I, the no K-correction case corresponds to a K-correction assuming a power-law 
Table 5. The full binned SXLF values -3

\begin{tabular}{|c|c|c|c|c|}
\hline \multicolumn{5}{|c|}{$\overline{\left(\Omega_{\mathrm{m}}, \Omega_{\Lambda}\right)}=(0.3,0.7)$} \\
\hline $\begin{array}{c}z \\
(1) \\
\end{array}$ & $\begin{array}{c}\log L_{\mathrm{x}}{ }^{\mathrm{a}} \\
(2)\end{array}$ & $\begin{array}{c}N^{\text {obs }} \\
(3) \\
\end{array}$ & $\begin{array}{c}N^{\mathrm{mdl}} \\
(4) \\
\end{array}$ & $\frac{\mathrm{d} \Phi^{\mathrm{n}}}{\mathrm{d} L_{\mathrm{x}} L_{\mathrm{x}}}$ \\
\hline $.015-0.2$ & $41.30-41.70$ & $5(3)$ & 1.0 & $\left(2.3_{-1.0}^{+1.4}\right) 10^{-4}$ \\
\hline $.015-0.2$ & $41.70-42.60$ & $12(3)$ & 10.6 & $\left(2.0_{-0.6}^{+0.7}\right) 10^{-5}$ \\
\hline $.015-0.2$ & $42.60-43.00$ & $19(2)$ & 20.5 & $\left(6.4_{-1.5}^{+1.8}\right) 10^{-6}$ \\
\hline $.015-0.2$ & $43.00-43.50$ & $58(6)$ & 53.5 & $\left(3.6_{-0.5}^{+0.5}\right) 10^{-6}$ \\
\hline $.015-0.2$ & $43.50-43.80$ & $49(3)$ & 49.1 & $\left(1.4_{-0.2}^{+0.2}\right) 10^{-6}$ \\
\hline $.015-0.2$ & $43.80-44.20$ & $62(5)$ & 67.5 & $\left(4.3_{-0.6}^{+0.6}\right) 10^{-7}$ \\
\hline $.015-0.2$ & $44.20-44.60$ & $41(1)$ & 41.8 & $\left(9.1_{-1.4}^{+1.6}\right) 10^{-8}$ \\
\hline $.015-0.2$ & $44.60-45.10$ & 27 & 21.9 & $\left(1.4_{-0.3}^{+0.3}\right) 10^{-8}$ \\
\hline $.015-0.2$ & $45.10-45.80$ & 1 & 2.5 & $\left(2.5_{-2.1}^{+5.1}\right) 10^{-10}$ \\
\hline $0.2-0.4$ & $41.70-42.20$ & 1 & 0.8 & $\left(7.8_{-6.5}^{+16}\right) 10^{-5}$ \\
\hline $0.2-0.4$ & $42.20-42.80$ & $2(1)$ & 4.5 & $\left(1.2_{-0.8}^{+1.4}\right) 10^{-5}$ \\
\hline $0.2-0.4$ & $42.80-43.40$ & $19(6)$ & 14.9 & $\left(1.3_{-0.3}^{+0.4}\right) 10^{-5}$ \\
\hline $0.2-0.4$ & $43.40-43.80$ & $21(2)$ & 21.3 & $\left(4.0_{-0.9}^{+1.0}\right) 10^{-6}$ \\
\hline $0.2-0.4$ & $43.80-44.30$ & $26(3)$ & 28.2 & $\left(1.1_{-0.2}^{+0.2}\right) 10^{-6}$ \\
\hline $0.2-0.4$ & $44.30-44.70$ & $17(2)$ & 19.8 & $\left(1.3_{-0.3}^{+0.4}\right) 10^{-7}$ \\
\hline $0.2-0.4$ & $44.70-45.30$ & 23 & 16.6 & $\left(1.5_{-0.3}^{+0.4}\right) 10^{-8}$ \\
\hline $0.2-0.4$ & $45.30-46.00$ & 4 & 5.7 & $\left(2.0_{-1.0}^{+0.5}\right) 10^{-10}$ \\
\hline $0.4-0.8$ & $42.20-42.80$ & 0 & 0.8 & $<6.810^{-5}$ \\
\hline 0.4 & $42.80-43.25$ & $5(3)$ & 5.5 & $\left(1.3_{-06}^{+0.8}\right) 10^{-5}$ \\
\hline $0.4-$ & $43.25-43.60$ & $13(7)$ & 9.8 & $\left(1.2_{-0.3}^{+0.4}\right) 10^{-5}$ \\
\hline $0.4-0.8$ & $43.60-44.10$ & $29(1)$ & 30.3 & $\left(4.0_{-0.7}^{+0.9}\right) 10^{-6}$ \\
\hline $0.4-0.8$ & $44.10-44.80$ & 31 & 34.0 & $\left(3.7_{-0.7}^{+0.8}\right) 10^{-7}$ \\
\hline $0.4-0.8$ & $44.80-45.40$ & $15(1)$ & 11.1 & $\left(2.1_{-0.5}^{+0.7}\right) 10^{-8}$ \\
\hline $0.4-($ & $45.40-46.00$ & 5 & 5.8 & $\left(6.1_{-2.6}^{+3.8}\right) 10^{-10}$ \\
\hline $0.4-0.8$ & $46.00-46.61$ & 1 & 2.5 & $\left(1.2_{-1.0}^{+2.6}\right) 10^{-11}$ \\
\hline & 60 & 6 & 3.9 & $\left(2.5_{-1.0}^{+1.4}\right) 10^{-5}$ \\
\hline & $43.60-44.10$ & $19(2)$ & 22.5 & $\left(7.9_{-1.8}^{+1.2}\right) 10^{-6}$ \\
\hline 0.8 & $44.10-44.60$ & 44 & 41.4 & $\left(3.8_{-0.6}^{+0.7}\right) 10^{-6}$ \\
\hline $0.8-1.6$ & $44.60-45.30$ & 51 & 47.7 & $\left(3.2_{-0.4}^{+0.5}\right) 10^{-7}$ \\
\hline $0.8-1.6$ & $45.30-46.00$ & 7 & 10.7 & $\left(4.5_{-1.7}^{+2.4}\right) 10^{-9}$ \\
\hline & $46.00-46.50$ & 4 & 3.2 & $\left(3.2_{-1.5}^{+2.3}\right) 10^{-10}$ \\
\hline $0.8-1.6$ & $46.50-47.00$ & 4 & 1.9 & $\left(3.5_{-1.7}^{+2.6}\right) 10^{-11}$ \\
\hline & 4 & 9 & 14.4 & $\left(5.6_{-1.8}^{+2.4}\right) 10^{-6}$ \\
\hline & $44.50-45.20$ & 18 & 15.0 & $\left(1.1_{-0.3}^{+0.3}\right) 10^{-6}$ \\
\hline $1.6-2.3$ & $45.20-45.90$ & 14 & 8.9 & $\left(5.2_{-14}^{+1.7}\right) 10^{-8}$ \\
\hline $1.6-2.3$ & $45.90-46.60$ & 2 & 3.0 & $\left(7.7_{-4.9}^{+9.2}\right) 10^{-10}$ \\
\hline $1.6-2.3$ & $46.60-47.00$ & 0 & 0.6 & $<2.910^{-10}$ \\
\hline $1.6-2.3$ & $47.00-47.63$ & 1 & 1.6 & $\left(4.4_{-3.6}^{+9.1}\right) 10^{-12}$ \\
\hline $2.3-4.6$ & -44.80 & 7 & 9.0 & $\left(4.6_{-1.7}^{+2.3}\right) 10^{-6}$ \\
\hline $2.3-4.6$ & $44.80-45.40$ & 8 & 6.6 & $\left(4.1_{-1.4}^{+1.9}\right) 10^{-7}$ \\
\hline $2.3-4.6$ & $45.40-46.20$ & 7 & 5.8 & $\left(1.9_{-0.7}^{+0.4}\right) 10^{-8}$ \\
\hline $2.3-4.6$ & $46.20-47.00$ & 1 & 2.2 & $\left(2.1_{-1.7}^{+4.4}\right) 10^{-10}$ \\
\hline $2.3-4.6$ & $47.00-47.63$ & 2 & 1.3 & $\left(3.0_{-2.0}^{+3.7}\right) 10^{-11}$ \\
\hline
\end{tabular}

See notes to Table 3.

photon index of $\Gamma=2$, which is the most representative spectrum for the soft X-ray sources in the sample. Thus for many purposes, considering our tabulated values as K-corrected SXLF would be accurate enough.
- Large-Scale Structure: The lowest redshift bin covers $0.015<z<0.2$ and there is some concern about the effect of the large-scale structure of the universe, which could be confused with the effect of evolution. Zucca et al. (1997) found an underdensity of galaxies in the local universe out to $z \sim 0.05$. However, it might be because of the structure within their survey field of $\sim 27\left[\mathrm{deg}^{2}\right]$ rather than that of the entire space out to this redshift. The solid angle surveyed by RBS is $\sim 50 \%$ of the sky and the fields of SA-N are scattered in various directions. In any event, our lowest redshift bin samples a sufficiently large volume of space to $z=0.2$ with a uniform redshift coverage, thus it is unlikely that the calculated SXLF is significantly biased by the large-scale structure of the universe.

Acknowledgements. This work is based on a combination of extensive ROSAT surveys from a number of groups. Our work is indebted to the effort of the ROSAT team and the optical followup teams in producing data and the catalogs used in the analysis. In particular, we thank K. Mason, A. Schwope, G. Zamorani, I. Appenzeller, and I. McHardy for providing us with and allowing us to use their data prior to publication of the catalogs. TM was supported by a fellowship from the Max-Planck-Society during his appointment at MPE. GH acknowledges DLR grant FKZ 50 OR 9403 5. We thank the referee, T. Shanks, for useful comments.

\section{References}

Appenzeller, I., Thiering, I., Zickgraf, F.-J., et al. 1998, ApJS, 117,319

Avni, Y., \& Bahcall, J. N. 1980, ApJ, 235, 694

Bower, R. G., Hasinger, G., Castander, F. J., et al. 1996, MNRAS, 281, 59

Boyle, B. J., Shanks, T., Georgantopoulos, I., Stewart, G. C., \& Griffiths, R. E. 1994, MNRAS, 271, 639

Comastri, A., Setti, G., Zamorani, G., \& Hasinger, G. 1995, A\&A, 296, 1

Fasano, G., \& Franceschini, A. 1987, MNRAS, 225, 155

Fischer, J.-U., Hasinger, G., Schwope, A. D., et al. 1998, Astron. Nachr., 319, 347

Gehrels, N. 1986, ApJ, 303, 336

Gilli, R., Risalti, G. \& Salvati, M. 1999, A\&A, 347, 424

Gilli, R., Salvati, M., \& Hasinger, G. 2001, A\&A, in press [astro-ph/0011341]

Hasinger, G. 1996, A\&AS, 120, C607

Hasinger, G. 1998, AN, 319, 37

Hasinger, G., Burg, R., Giacconi, R., et al. 1993, A\&A, 275, 1

Hasinger, G., Burg, R., Giacconi, R., et al. 1998, A\&A, 329, 482

Hasinger, G., Lehmann, I., Giacconi, R., et al. 1999, in Highlights in X-ray Astronomy in Honor of Joachim Trümper's 65th Birthday, MPE Report (Garching: MPE)

Hasinger, G., Altieri, B., Arnaud, M., et al. 2001, A\&A, 365, L45

Hornschemeier, A. E., Brandt, W. N., Garmire, G. P., et al., ApJ, in press [astro/ph-0042460]

Jones, L. R., McHardy, I. M., Merrifield, M. R., et al. 1996, MNRAS, 285, 547

Lehmann, I., Hasinger, G., Schmidt, M., et al. 2000, A\&A, 352, 35 
Madau, P., Ghisellini, G., \& Fabian, A. C. 1994, MNRAS, 270, L17

Marshall, H. L., Avni, Y., Tananbaum, H., \& Zamorani, G. 1983, ApJ, 269, 35

Mason, K., Carrera, F. J., Hasinger, G., et al. 2000, MNRAS, 311,456

McHardy, I., Jones, L. R., Merrifield, M. R., et al. 1998, MNRAS, 295, 641

Miyaji, T., Ishisaki, Y., Ogasaka, Y., Ueda, Y., et al. 1998, A\&A, 334, L13

Miyaji, T., Hasinger, G., \& Schmidt, M. 1999, in Highlights in X-ray Astronomy in Honor of Joachim Trümper's 65th Birthday, MPE Report (Garching: MPE), in press [astro-ph/9809398], M99a

Miyaji, T., Hasinger, G., \& Schmidt, M. 2000, A\&A, 353, 25, Paper I

Miyaji, T., Hasinger, G., \& Schmidt, M. 2000, Adv. Sp. Res., 25,827

Mushotzky, R. F., Cowie, L. L., Barger, A. J., \& Arnaud, K. A. 2000, Nature, 404, 459

Page, M. J., Mason, K. O., McHardy, I. M., Jones, L. R., \& Carrera, F. J. 1997, MNRAS, 291, 324
Page, M. J., \& Carrera, F. J. 1999, MNRAS, 311, 433

Press, W. H., Teukolsky, S. A., Vetterling, W. T., \& Flannery, B. P. 1992 Numerical Recipes in Fortran (Cambridge: Cambridge Univ. Press), 640

Schmidt, M. 1968, ApJ, 151, 393

Schmidt, M, \& Green, R. F. 1983, ApJ, 269, 352 the Universe, MPE Report 263 (Garching: MPE), 395

Schmidt, M., Hasinger, G., Gunn, J., et al. 1998, A\&A, 329, 495

Schwope, A. D., Hasinger, G., Lehmann, I., et al. 2000, Astr. Nachr., 321, 1

Voges, W. 1994, in Basic Space Science, ed. H. J. Haubold, \& L. I. Onuora, 202

Voges, W., Aschenbach, B., Boller, Th., et al. 1999, A\&A, 349, 389

Wisotzki, L. 1998, Astr. Nachr., 319, 257

Zamorani, G., Mignoli, M, Hasinger, G., et al. 1999, A\&AS, 346,732

Zickgraf, F.-J., Thiering, I., Krautter, J., et al. 1997, A\&AS, 123, 103

Zucca, E., Zamorani, G., Vettolani, G., et al. 1997, A\&A, 326, 477 